\title{
Percutaneous endovascular therapy for symptomatic chronic total occlusion of the left subclavian artery
}

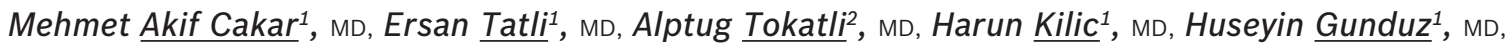 \\ Ramazan Akdemir $^{1}$, MD
}

INTRODUCTION Percutaneous endovascular therapy is an accepted and preferred procedure for symptomatic subclavian artery disease. However, the technical feasibility and effectiveness of treating chronic total occlusion of the subclavian artery with this approach is uncertain. We aimed to evaluate the initial and mid-term results of endovascular therapy for patients with symptomatic chronic total occlusion of the left subclavian artery.

METHODS Consecutive patients who underwent balloon angioplasty and stenting for chronic total occlusion of the left subclavian artery between January 2010 and February 2014 were included.

RESULTS Overall, 16 patients (10 male, 6 female; mean age $56 \pm 13$ years) underwent balloon angioplasty and stenting for chronic total occlusion of the left subclavian artery. 6 (37.5\%) had arm claudication, 8 (50.0\%) had vertebrobasilar insufficiency and 2 (12.5\%) had coronary steal. 18 balloon-expandable stents were implanted in 15 patients. Central luminal passage was not achieved in one patient because of the subintimal position of the guidewire (procedural success rate $93.8 \%)$. There were no procedure-related complications. Mean preprocedural and postprocedural systolic blood pressure differences between the upper extremities were $37 \pm 13$ (range 25-60) $\mathrm{mmHg}$ and $11 \pm 9$ (range 5-38) $\mathrm{mmHg}$, respectively; the improvement was statistically significant. Outpatient follow-up revealed one asymptomatic restenosis at two years. The patency rate at two years was $93.3 \%$.

CONCLUSION Balloon angioplasty and stenting for chronic total occlusion of the left subclavian artery is safe and effective, with good acute success rate and mid-term patency. Prospective randomised studies on larger patient populations would provide more precise results.

Keywords: angioplasty, stenting, subclavian artery, total occlusion

\section{INTRODUCTION}

Percutaneous transluminal balloon angioplasty (PTA) and stenting is an accepted treatment choice for subclavian artery (SA) occlusions. ${ }^{(1)}$ Chronic total occlusion (CTO) of the SA is defined as complete occlusion of the SA, with no distal flow, that is present for an estimated duration of three months. CTO of the SA is rarely encountered and its precipitating factors are similar to those of atherosclerosis (e.g. smoking, diabetes mellitus, hypertension and hypercholesterolaemia). ${ }^{(2)}$ Other causes include arteritis, compression of a mass and associated congenital heart disease. ${ }^{(3)}$ Arm claudication is the most common symptom of SA stenosis. ${ }^{(4)}$ Subclavian steal syndrome, which is associated with vertebrobasilar insufficiency, is another possible condition (Fig. 1). Furthermore, SA occlusion may lead to coronary steal syndrome in patients with prior internal mammary coronary bypass surgery. ${ }^{(5)}$

Although revascularisation of subclavian occlusion in patients with symptomatic subclavian steal, carotid steal, arm claudication or coronary steal has been the traditional surgical approach, ${ }^{(6)}$ with the evolution of endovascular techniques, SA occlusions can now be treated with balloon angioplasty and stenting. ${ }^{(7)}$ The present study was undertaken to examine the technical feasibility, effectiveness and reliability of treating CTO of the left SA, as well as to determine the mid-term results of PTA and stenting, through a retrospective review of a series of 16 consecutive patients who had total occlusion of the left SA.

\section{METHODS}

A single-centre retrospective study was conducted with 16 consecutive symptomatic patients who were diagnosed with CTO of the left SA between January 2010 and February 2014. Chronicity of the CTO was assessed based on the duration of symptoms and imaging documentation. We included all patients who were symptomatic and underwent baseline colour duplex ultrasonography and/or angiography, which showed total occlusion of the SA.

Informed consent from the patients and approval from the institutional review board were obtained. Preprocedural evaluations included patients' baseline characteristics, careful history-taking and physical examination. Technical and clinical determinants vis-à-vis the procedure, complications and followup were analysed using the institutional database.

All procedures were performed in the cardiac catheterisation laboratory and under local anaesthesia. All patients were receiving antiplatelet therapy with acetyl salicylic acid at the time of diagnosis (dose range 75-325 mg/day). In addition, clopidogrel was given orally (dose $75 \mathrm{mg} /$ day) for at least one week before the procedure or administered as a loading dose (dose $300 \mathrm{mg}$ ) on the morning of the procedure. The dual antiplatelet therapy was maintained for four weeks.

During the procedure, an 8-F sheath was used for the right femoral artery, whereas a $6-\mathrm{F}$ introducer was used for the left 

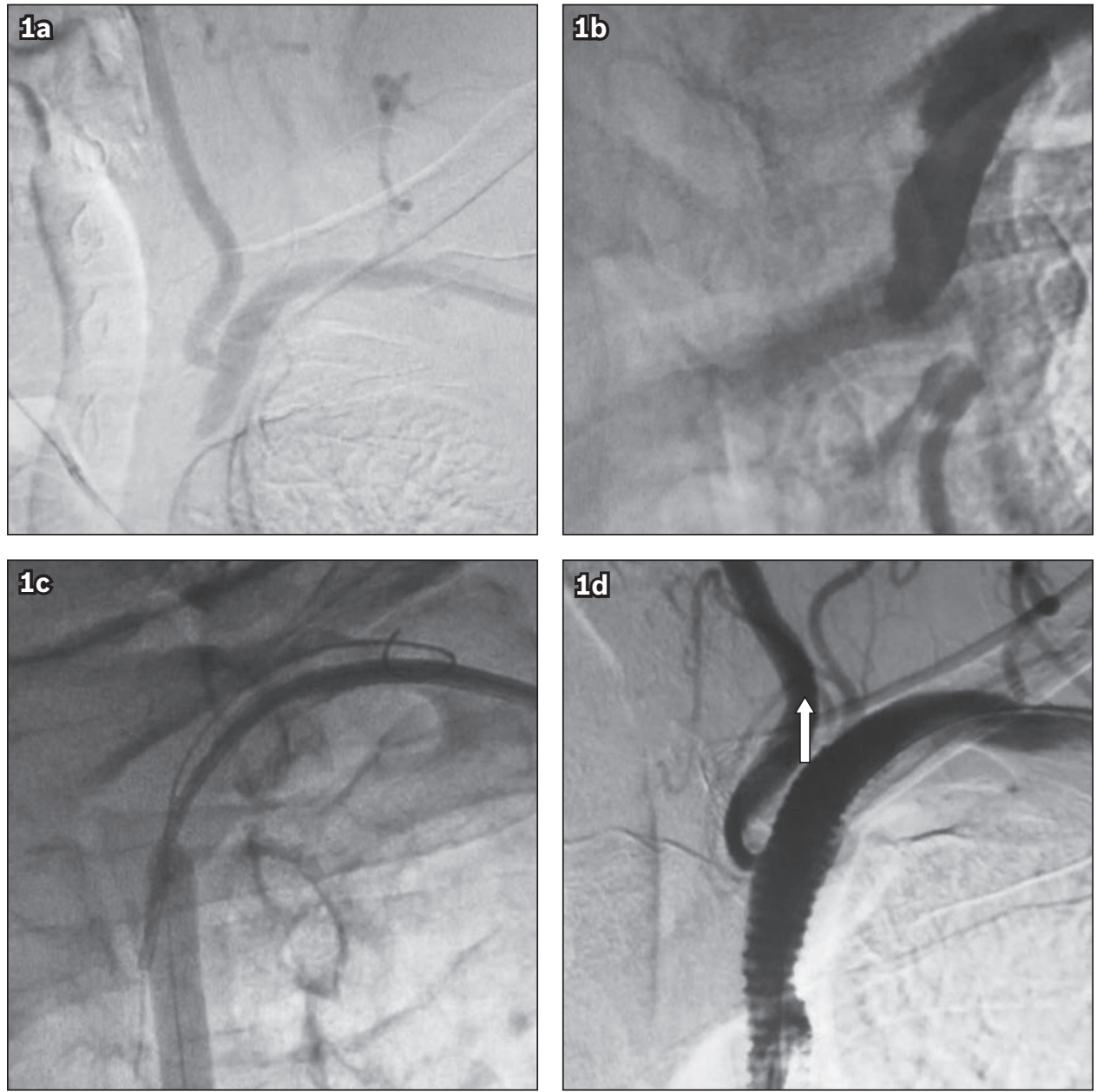

Fig. 1 Angiograms show (a) subclavian steal syndrome; (b) total occlusion of the proximal left subclavian artery; (c) percutaneous transluminal balloon angioplasty; and (d) stent deployment and anterograde flow in the left vertebral artery (arrow).

brachial artery. In patients with coronary steal, a 6-F sheath was advanced to the femoral artery, and a left Judkins diagnostic catheter that engaged the left coronary artery ostia was subsequently introduced over this sheath. Left coronary dye injection filled the distal part of the left SA due to steal, confirming the distal part of the left SA. This was used as guidance for intervention (Figs. $2 \& 3$ ). During all procedures for totally occluded SAs, an 8-F sheath was advanced over the 7-F guiding internal mammary artery catheter to the stump of the left SA. Subsequently, a 0.035-inch hydrophiliccoated guidewire (Glidewire ${ }^{\circledR}$; Medi-tech/Boston Scientific Corporation, Natick, MA, USA) was used to cross the CTO. If the guidewire could not cross the lesion, a 0.018-inch Treasure wire (Asahi Intecc Co, Aichi, Japan) or CTO-dedicated wires were used. The guidewire was advanced to the brachial artery after crossing the lesion and the guiding catheter was introduced over the wire (Fig. 4). Predilation was performed using an undersized PTA balloon (4-6 mm × 40-60 mm; GliderfleX®; TriReme Medical, Pleasanton, CA, USA) (Fig. 5). Peripheral balloon-expanding stents (size 6-9 mm × 18-38 mm; Qualimed Propes) were used (Fig. 6). Aspirin (dose $300 \mathrm{mg} /$ day) and clopidogrel (dose $75 \mathrm{mg} /$ day) were continued after intervention.

Central luminal passage was not achieved in one patient because of the subintimal position of the guidewire. Angiographic control was performed after the procedure. Technical success was defined as residual stenosis of over $30 \%$ and clinical success was defined as technical success with resolution of symptoms.

All patients were followed up at one month, six months and 24 months after the procedure. At follow-up, in addition to history and physical examination, duplex ultrasonography was also performed. Restenosis was defined as recurrent symptoms accompanied by recurrent stenosis of over $60 \%$, as confirmed by duplex ultrasonography.

Continuous variables were presented as mean \pm standard deviation or number and percentage. For categorical and continuous variables, chi-square test and Student's $t$-test were used, respectively. A p-value $<0.05$ was considered to be statistically significant. All statistical analyses were performed using SPSS version 16.0 (SPSS Inc, Chicago, IL, USA).

\section{RESULTS}

Overall, 16 patients with CTO of the left SA met the inclusion criteria. In our study, the clinical indications for percutaneous intervention were arm claudication for $6(37.5 \%)$ patients, vertebrobasilar insufficiency for $8(50.0 \%)$ patients and coronary steal for 2 (12.5\%) patients. Patients had some comorbidities and risk factors, such as coronary artery disease $(31.3 \%)$, hyperlipidaemia (75.0\%), hypertension $(68.8 \%)$, diabetes mellitus $(43.8 \%)$ and smoking (75.0\%); 1 (6.3\%) patient had renal insufficiency (Table I). 

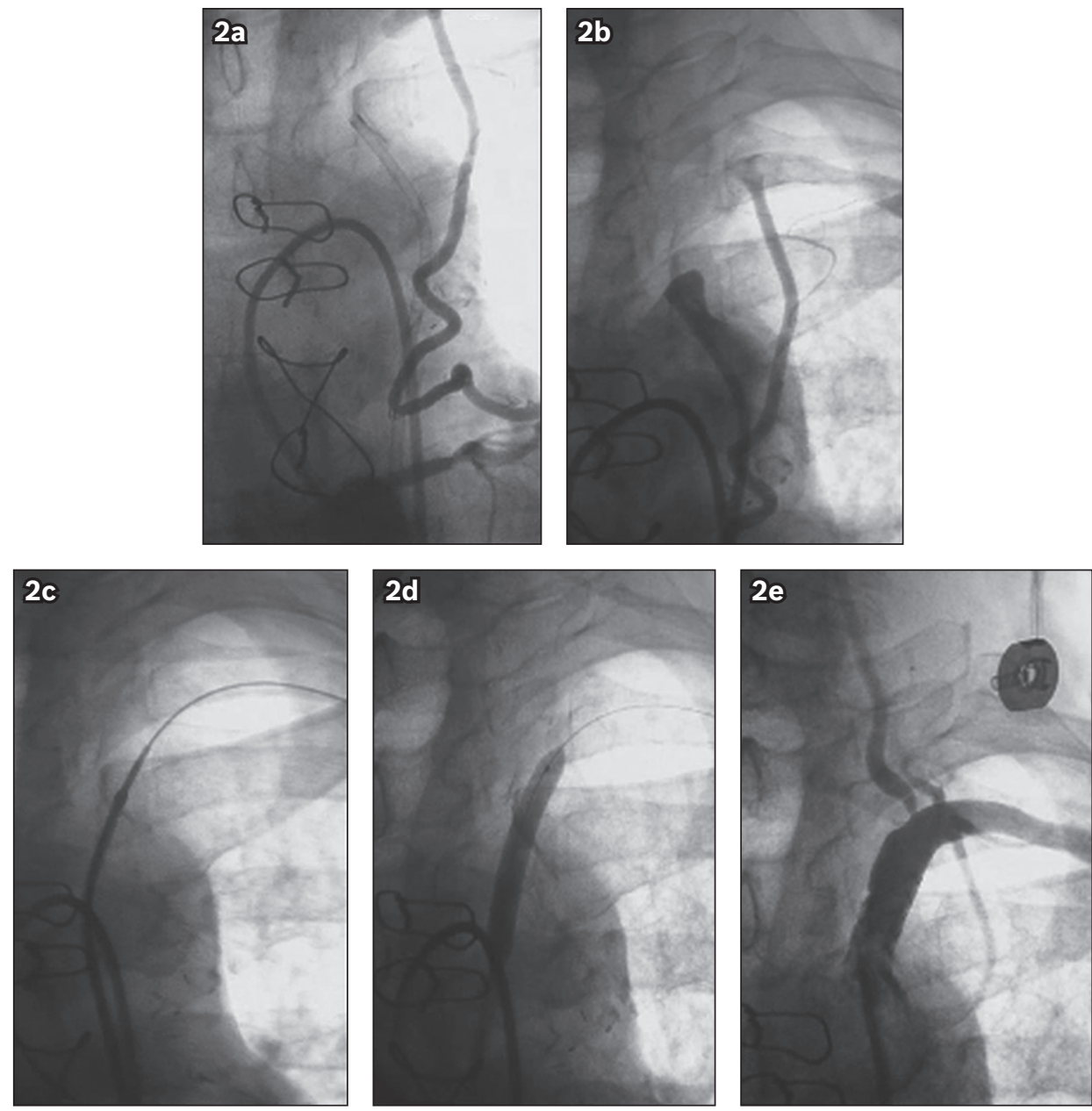

Fig. 2 Angiograms show (a) retrograde flow in the internal mammary artery with filling of the left subclavian arteries; (b) total occlusion in the ostial lesion of the left subclavian artery; (c) lesion crossing with the wire; (d) predilation during balloon angioplasty; and (e) post-angioplasty control, with the stent implant showing good patency of the left subclavian artery and maintenance of the vertebral and internal mammary arteries.

Successful percutaneous angioplasty and stenting was achieved for 15 (93.8\%) patients, but percutaneous intervention failed for $1(6.3 \%)$ patient with arm claudication because of an inability to cross the lesion with wire despite multiple attempts. Consequently, the rate of acute technical success for the entire group was $93.8 \%$. Among the 15 patients with successful balloon angioplasty and stenting, 18 balloon-expandable stents were implanted (mean length $28.6 \pm 11.4 \mathrm{~mm}$; mean diameter $7.4 \pm$ $2.2 \mathrm{~mm}$ ). A single stent was placed in 12 patients, while three patients received two stents each.

Access site complications, neurologic events, distal embolisation or death was not encountered during the periprocedural period in any of our patients. The mean preprocedural systolic blood pressure difference between the upper extremities was $37 \pm 13$ (range 25-60) $\mathrm{mmHg}$, while the corresponding postprocedural difference was $11 \pm 9$ (range 5-38) $\mathrm{mmHg}$. The improvement following the intervention was statistically significant $(\mathrm{p}<0.05)$. During the follow-up period, restenosis only occurred in one patient, who was asymptomatic and was treated with exercise rehabilitation and medical therapy. Among the remaining 14 patients, no complications or restenosis were observed at 24 months (Table II), resulting in a patency rate of $93.3 \%$.

\section{DISCUSSION}

Although the conventional treatment of SA occlusion involves surgical procedures with good outcomes, many serious complications have been reported, and associated mortality and morbidity remain high. ${ }^{(8)}$ Due to such reasons, treatment of subclavian stenosis has shifted towards percutaneous procedures during the last decade. However, controversy persists regarding the safety, feasibility and outcomes of percutaneous intervention for CTO of the SA. ${ }^{(9)}$

In the present study, there were no minor or major complications with balloon angioplasty and stenting in patients with CTO of the SA. Our technical success rate was $93.8 \%$ and restenosis occurred in only one patient during 24 months of follow-up. Previous reports have also showed high rates of technical success and low complication rates. ${ }^{(10)}$

Symptoms are the main indication for percutaneous intervention for CTO of the SA. In our study, the most common indications were vertebrobasilar insufficiency $(n=8,50.0 \%)$, arm claudication ( $n=6,37.5 \%)$ and coronary steal $(n=2,12.5 \%)$. Revascularisation options for symptomatic CTO of the SA include percutaneous and surgical treatments. During surgery, a variety of bypass techniques can be performed, such as carotid-subclavian bypass, carotid-subclavian transposition, and axillo-axillary or 
Table I. Demographic and clinical characteristics of patients who underwent subclavian stenting $(n=16)$.

\begin{tabular}{|ll|}
\hline Variable & No. $(\%) /$ mean \pm SD \\
\hline Age $(\mathbf{y r})$ & $56.2 \pm 12.8$ \\
\hline Male gender & $10(62.5)$ \\
\hline Symptom & $6(37.5)$ \\
\hline Arm claudication & $8(50.0)$ \\
\hline Vertebrobasilar insufficiency & $2(12.5)$ \\
\hline Coronary steal & \\
\hline Risk factor & $5(31.3)$ \\
\hline Coronary artery disease & $12(75.0)$ \\
\hline Hyperlipidaemia & $11(68.8)$ \\
\hline Hypertension & $7(43.8)$ \\
\hline Diabetes mellitus & $12(75.0)$ \\
\hline Smoking & $1(6.3)$ \\
\hline Renal insufficiency & \\
\hline
\end{tabular}

SD: standard deviation

Table II. Procedural data and result of subclavian artery BA and stenting at initial and two-year follow-up $(n=16)$.

\begin{tabular}{|ll|}
\hline Variable & No. $(\%) /$ mean \pm SD \\
\hline Stent length $(\mathrm{mm})$ & $28.6 \pm 11.4$ \\
\hline Stent diameter $(\mathrm{mm})$ & $7.4 \pm 2.2$ \\
\hline Successful BA and stenting & $15(93.8)$ \\
\hline Unsuccessful BA and stenting & $1(6.3)$ \\
\hline Procedural complication (major/minor) & $0(0)$ \\
\hline Total restenosis (occlusion) at 2 yr & $1(6.3)$ \\
\hline
\end{tabular}

BA: balloon angioplasty; SD: standard deviation

subclavian-subclavian bypass. These approaches have been shown to have good long-term patency rates (range $82 \%-100 \%$ ). ${ }^{11,12}$ However, extensive dissection and manipulation during the surgery can result in serious injuries to adjacent tissues. Thus, mortality rates for surgery can be as high as $10 \%$, whereas related morbidity can be nearly $25 \% .{ }^{(13)}$ Even with extrathoracic techniques, mortality rates remain in the range of $2 \%-5 \% .{ }^{(14)}$

Major surgical complications include injuries of the brachial plexus, vagal nerve, laryngeal nerve and phrenic nerve. Cerebral ischaemia, Horner syndrome and chylothorax are other main complications. ${ }^{(13)}$ Qi et al evaluated the surgical treatment of SA occlusion in a series involving 39 patients. In their study, although the initial and long-term patency rates were very good, the complication rate was $13.5 \% .{ }^{(15)}$ Schardey et al also found similar results in a series of subclavian carotid transposition for subclavian occlusion treatment and reported a complication rate of $15 \% .{ }^{(16)}$ It is difficult to express an opinion about the comparison between surgical and endovascular approaches for patients with subclavian occlusion because of a lack of randomised studies. Still, endovascular treatment for symptomatic occlusive disease of the SA has increasingly become the preferred option and is suggested as the first line of treatment. ${ }^{(17)}$

Balloon angioplasty alone for the treatment of cervical occlusive arterial disease was introduced in the 1980s. ${ }^{(18)}$
However, when used alone for CTO, balloon angioplasty was found to be less effective. ${ }^{(19)}$ In studies involving series of patients undergoing pure balloon angioplasty, patency rates have been around $50 \%$ or as low as $15 \%$ at follow-up. ${ }^{(20)}$ Comparing the patency rates for SA stenosis between a group that had PTA with stenting and another group that had balloon angioplasty alone, Henry et al found significantly better results among the stenting group ${ }^{(6)}$ Sixt et al also recommended stenting for CTO of the SA. ${ }^{(21)}$ In contrast to balloon angioplasty alone, stenting for CTO of the SA has provided durable outcomes. ${ }^{(22)}$ With the use of stents, procedural success rates for CTO were shown to increase to $70 \%-100 \% .{ }^{(23)}$ In our cohort, a combination of balloon angioplasty and stenting was achieved for all patients with the use of balloon-expandable stents.

Some critical anatomical details must be considered, including the relationship of the stenosis to the vertebral and left internal mammary arteries. ${ }^{(24)}$ Stenosis that is close to the origin of the vertebral artery may require complex interventions. This can lead to vertebral artery dissections and catastrophic complications. The left internal mammary artery is also in close proximity to the distal endpoint of the stent, and its origin must be addressed as well, so that its present or future use as a conduit for coronary bypass surgery is not hindered.

We used balloon-expandable stents with an emphasis on correct placement and high radial force. Balloon-expandable stents have been the main stents in many previous studies. ${ }^{(25)}$ They have a higher radial force and offer more precise placement in comparison to self-expanding stents. For ostial lesions, stent dislocation into the aorta may be provoked by self-expanding stents, and therefore balloon-expandable stents should be used while avoiding the use of self-expanding or undersized stents. If the lesion is non-ostial, the use of a self-expanding stent can be considered, with the knowledge that predilation and postdilation would likely be mandatory. As reported by Sixt et al, self-expanding stents support better long-term prevention of restenosis. $^{(21)}$

Recanalisation failure is common in CTO of the SA. This failure may be related to calcification, density and length of the occluded segment. ${ }^{(26)}$ In our study, all attempts to pass the lesion were unsuccessful in one patient. In such cases, aggressive efforts to traverse the lesion may produce severe complications. The other complications of PTA, such as stent dislocation, thrombosis, dissection and infection, were not seen in our study. In-stent restenosis is another concern with endovascular procedures. Some studies have found that short-term and mid-term results for in-stent restenosis, at least during the first two years, were very low. ${ }^{(27)}$ Although there is a paucity of long-term results, it has been shown that stenting for symptomatic SA stenosis had $83 \%$ primary and $96 \%$ secondary patency rates on long-term followup. ${ }^{(28)}$ In-stent restenosis can be treated with balloon angioplasty or repeat stenting for symptomatic patients. As our patient with restenosis remained symptom-free, we did not perform further interventions.

There were some limitations to our study. As this was a retrospective single-centre study with a small sample size, further 
extensive and prospective studies are warranted to corroborate our findings. Notwithstanding this, our series of patients with symptomatic CTO of the left SA who were treated with balloon angioplasty and stenting showed remarkable procedural success rate, satisfactory initial and mid-term patency rates, and a very low complication rate.

\section{REFERENCES}

1. Smith JM, Koury HI, Hafner CD, Welling RE. Subclavian steal syndrome. A review of 59 consecutive cases. J Cardiovasc Surg (Torino) 1994; 35:11-4.

2. Schillinger M, Haumer M, Schillinger S, Ahmadi R, Minar E. Risk stratification for subclavian artery angioplasty: is there an increased rate of restenosis after stent implantation? J Endovasc Ther 2001; 8:550-7.

3. Jaeger HJ, Mathias KD, Kempkes U. Bilateral subclavian steal syndrome: treatment with percutaneous transluminal angioplasty and stent placement. Cardiovasc Intervent Radiol 1994; 17:328-32.

4. van der Steen A, Geervliet JP. Collateral circulation and steal mechanisms in innominate and subclavian artery obstruction. Clin Neurol Neurosurg 1979; 81:194-8.

5. Labropoulos N, Nandivada P, Bekelis K. Prevalence and impact of the subclavian steal syndrome. Ann Surg 2010; 252:166-70.

6. Henry M, Henry I, Polydorou A, Polydorou A, Hugel M. Percutaneous transluminal angioplasty of the subclavian arteries. Int Angiol 2007; 26:324-40.

7. Müller-Hülsbeck S. Subclavian and vertebral arterial interventions. Semin Intervent Radiol 2007; 24:258-67.

8. AbuRahma AF, Robinson PA and Jennings TG. Carotid-subclavian artery bypass grafting with polytetrafluoroethylene grafts for symptomatic subclavian artery stenosis or occlusion: a 20-year experience. J Vasc Surg 2000; 32:411-9.

9. Mathias KD, Luth I, Haarmann P. Percutaneous transluminal angioplasty of proximal subclavian artery occlusions. Cardiovasc Intervent Radiol 1993; 16:214-8.

10. Sullivan TM, Gray BH, Bacharach JM, et al. Angioplasty and primary stenting of the subclavian, innominate, and common carotid arteries in 83 patients. J Vasc Surg 1998; 28:1059-65.

11. Ballotta E, Da Giau G, Abbruzzese E, et al. Subclavian carotid transposition for symptomatic subclavian artery stenosis or occlusion. A comparison with the endovascular procedure. Int Angiol 2002; 21:138-44.

12. Mingoli A, Sapienza P, Feldhaus RJ, et al. Long-term results and outcomes of crossover axilloaxillary bypass grafting: a 24-year experience. J Vasc Surg 1999;
29:894-901.

13. Zaytsev AY, Stoyda AY, Smirnov VE, et al. Endovascular treatment of supra-aortic extracranial stenoses in patients with vertebrobasilar insufficiency symptoms. Cardiovasc Intervent Radiol 2006; 29:731-8.

14. Queral LA. Endovascular treatment of focal aortic arch branch lesions. Ann Vasc Surg 1998; 12:396-403.

15. Qi L, Gu Y, Zhang J, et al. [Surgical treatment of subclavian artery occlusion]. Zhongguo Xiu Fu Chong Jian Wai Ke Za Zhi 2010; 24:1030-2. Chinese.

16. Schardey HM, Meyer G, Rau HG, et al. Subclavian carotid transposition: an analysis of a clinical series and a review of the literature. Eur J Vasc Endovasc Surg 1996; 12:431-6.

17. Babic S, Sagic D, Radak D, et al. Initial and long-term results of endovascular therapy for chronic total occlusion of the subclavian artery. Cardiovasc Intervent Radiol 2012; 35:255-62.

18. Farina C, Mingoli A, Schultz RD, et al. Percutaneous transluminal angioplasty versus surgery for subclavian artery occlusive disease. Am J Surg 1989; 158:511-4.

19. Hebrang A, Maskovic J, Tomac B. Percutaneous transluminal angioplasty of the subclavian arteries: long-term results in 52 patients. AJR Am J Roentgenol $1991 ; 156: 1091-4$

20. Motarjeme A, Gordon GI. Percutaneous transluminal angioplasty of the brachiocephalic vessels: guidelines for therapy. Int Angiol 1993; 12:260-9.

21. Sixt S, Rastan A, Schwarzwälder U, et al. Results after balloon angioplasty or stenting of atherosclerotic subclavian artery obstruction. Catheter Cardiovasc Interv 2009; 73:395-403.

22. Rodriguez-Lopez JA, Soler L, Werner A, et al. Stenting for atherosclerotic occlusive disease of the subclavian artery. Ann Vasc Surg 1999; 13:254-60.

23. Kumar K, Dorros G, Bates MC, et al. Primary stent deployment in occlusive subclavian artery disease. Cathet Cardiovasc Diagn 1995; 34:281-5.

24. Amor M, Eid-Lidt G, Chati Z, Wilentz JR. Endovascular treatment of the subclavian artery: stent implantation with or without predilatation. Catheter Cardiovasc Interv 2004; 63:364-70.

25. De Vries JP, Jager LC, Van den Berg JC, et al. Durability of percutaneous transluminal angioplasty for obstructive lesions of proximal subclavian artery: long term results. J Vasc Surg 2005; 41:19-23.

26. de Souza JM, Espinosa G, Santos Machado M, Soares PJ. Bilateral occlusion associated to steal phenomenon of internal carotid and left subclavian arteries: treatment by angioplasty and stenting. Surg Neurol 2007; 67:298-302.

27. Sakai C, Sakai N, Kuroiwa T, et al. Stenting for chronic total occlusion of the proximal subclavian artery. Interv Neuroradiol 2007; 13:135-40.

28. Patel SN, White CJ, Collins TJ, et al. Catheter-based treatment of the subclavian and innominate arteries. Catheter Cardiovasc Interv 2008; 71:963-8. 